\title{
Structural studies of bacterial small alarmone hydrolases
}

\author{
A. Chrenková, F. Bisiak, and D. E. Brodersen \\ Department of Molecular Biology and Genetics, Aarhus University, DK-8000 Aarhus C, Denmark \\ adch@mbg.au.dk
}

The stringent response is a fundamental mechanism for bacterial survival and adaptation to a wide range of stress conditions, mediated by the synthesis and hydrolysis of signal molecules collectively referred to as alarmones [1,2]. Bifunctional enzymes belonging to the RelA-SpoT homologue (RSH) superfamily are responsible for the synthesis of alarmones guanosine 5'-triphosphate 3'-diphosphate (pppGpp), guanosine 5'-diphosphate 3'-diphosphate (ppGpp), and guanosine 5'-monophosphate 3'-diphosphate (pGpp), and contain both synthetase and hydrolase domains, however, with the hydrolase domain being inactive in many cases. Some organisms additionally contain monofunctional small alarmone synthetases (SAS) and small alarmone hydrolases (SAH), regulation of which has not yet been fully described [3].

Here, we present a $1.2 \AA$ structure of the Leptospira levetii small alarmone hydrolase (SAH) and a $1.8 \AA$ structure of Corynebacterium glutamicum small alarmone hydrolase, together with an analysis of similarities and differences with the known bifunctional Rel enzyme structures. We show that the SAH structures contain common features typical for hydrolases, such as a metal ion binding site and the highly conserved histidine-aspartate (HD) motif. Analysis of the structures using PISA surprisingly revealed they both form dimers, in contrast to earlier reports. Moreover, there is a distinct difference in the dimerization interface, despite a high degree of structure conservation between the monomers. Dimer formation was confirmed experimentally using size exclusion chromatography multi-angle light scattering (SEC-MALS) and small angle X-ray scattering (SAXS). The structures of the two small alarmone hydrolase representatives allowed for a structural analysis and comparison with known bifunctional hydrolase structures (RelSeq from Streptococcus equisimilis and RelTh from Thermus thermophilus) [4, 5]. We demonstrate that while the key residues involved in substrate coordination are clearly conserved, there are some notable differences in several secondary structure elements. Intriguingly, we find differences in the position of helices involved in the regulation of activity in bifunctional hydrolases, that adopt an unanticipated position in the SAH structures. Taken together, our data shed new light on how small alarmone hydrolases are regulated as well as how they differ from the larger bifunctional enzymes. In the future, this will help us further understand the role of monofunctional SAH enzymes and in turn bring forward a better understanding of the stringent response in bacteria.

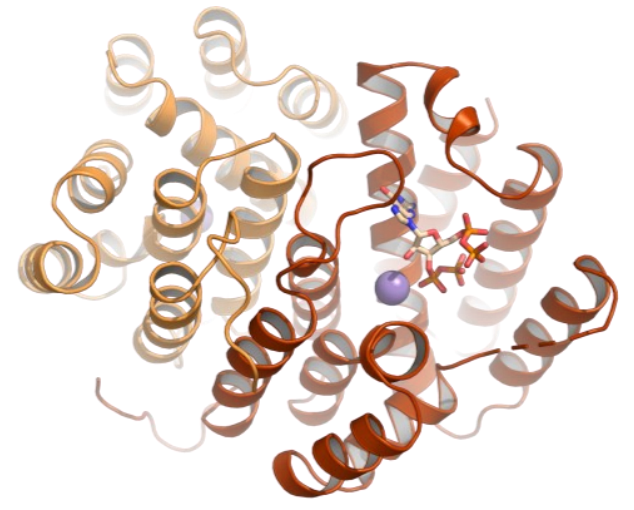

Figure 1. Structure of the Leptospira levetii SAH with a ppGpp molecule from RelSeq structure modelled in the active site.

[1] Irving, S. E., Choudhury, N. R., \& Corrigan, R. M. (2021). Nat Rev Microbiol, 19(4), 256-271.

[2] Cashel, M., \& Gallant, J. (1969). Nature, 221(5183).

[3] Atkinson, G. C., Tenson, T., \& Hauryliuk, V. (2011). PLoS One, 6(8), e23479.

[4] Hogg, T., Mechold, U., Malke, H., Cashel, M., \& Hilgenfeld, R. (2004). Cell, 117(1), 57-68.

[5] Tamman, H., Van Nerom, K., Takada, H., Vandenberk, N., Scholl, D., Polikanov, Y., Hofkens, J., Talavera, A., Hauryliuk, V., Hendrix, J., Garcia-Pino, A. (2020). Nat Chem Biol, 16(8), 834-840.

Keywords: stringent response; alarmones; stress response; X-ray crystallography 